\title{
ON A THEOREM OF HUNEKE CONCERNING MULTIPLICITIES
}

\author{
LIAM O'CARROLL
}

\begin{abstract}
We consider a theorem by Huneke on multiplicities, and show that the extension surmised by Huneke is a stronger form of the Syzygy Conjecture.
\end{abstract}

Craig Huneke has proved the following result.

THEOREM $1[9]$. Let $(A, \underline{m})$ be a complete local ring containing a field, let $n$ be a positive integer and let $e(A)$ be the multiplicity of $A$. Suppose that $A$ satisfies Serre's condition $S_{n}$ and that $e(A) \leq n$; if $n=1$ suppose further that $A$ is equidimensional. Then $A$ is Cohen-Macaulay.

All rings considered in this note are commutative, Noetherian and have an identity element, and all modules are finitely generated. For a discussion of the theory of (complete) local rings and of multiplicities, see $[\mathbf{1 1}, \mathbf{1 2}, \mathbf{1 3}, 14]$. A finitely generated module $M$ over a Noetherian $\operatorname{ring} A$ is said to satisfy $S_{n}$ if

$$
\operatorname{depth} M_{P} \geq \inf (n, \text { height } P) \text { for all } P \in \operatorname{Spec} A \text {. }
$$

As remarked on $\left[\mathbf{1 1}\right.$, p. 125], the condition $S_{1}$ is equivalent to $A$ having no embedded associated primes. We use the term 'equidimensional' in the sense of Grothendieck's E.G.A., i.e. a local ring is said to be equidimensional when all its minimal primes have the same co-height. Recall from [7, (5.10.9)] that a catenary (and so a complete) local ring which satisfies $S_{2}$ is equidimensional. A recent handy reference for much of the material in this note is the monograph [5], where a special case of Huneke's result is discussed on pp. 71-72 and on p. 75. As the latter shows, and as has been remarked by Huneke himself, in the case $n=1$ it is essential in Theorem 1 to add the hypothesis of equidimensionality.

From his theorem Huneke deduces results by Nagata [12, (40.6)] and by Ikeda, in the case where the rings involved contain a field. (As regards the former result, see the interesting aside on $[6$, p. 41].) Since these are known to hold without this restriction, Huneke surmises that the following is probably true:

Theorem 1 is valid without the assumption that $A$ contain a field.

Now recall the Syzygy Conjecture [4, p. 143] for regular local rings, which runs as follows:

if $R$ is a regular local ring, and if $K$ is the

jth syzygy of an $R$-module with $\operatorname{rank} K<j$, then $K$ is a free $R$-module.

Received by the editors November 4, 1985.

1980 Mathematics Subject Classification (1985 Revision). Primary 13H15.

Key words and phrases. Multiplicity, local ring, syzygy.

(C) 1987 American Mathematical Society $0002-9939 / 87 \$ 1.00+\$ .25$ per page 
For a discussion of the links between $j$ th syzygies and the condition $S_{j}$, and for a proof that (2) holds when $R$ contains a field, see [5, Chapter 3] and especially [loc. cit., Theorem 3.8 and Corollary 3.16]. It is also known that (2) holds when $\operatorname{dim} R \leq 4$ [4, p. 147].

Our final conjecture is the following:

Let $(R, \underline{m})$ be a regular local ring, let $\left(B, \underline{m}^{\prime}\right)$ be a local ring which dominates $[\mathbf{1 2}, p$. 14] $R$ and suppose that $B$ is a finitely generated $R$-module. Let $n$ be a positive integer. If $B$ satisfies $S_{n}$ and if the multiplicity $e_{R}(B) \leq n$ (where, if $n=1, B$ is also supposed to be equidimensional), then $B$ is Cohen-Macaulay.

REMARKS. Note that $B$ is catenary, being the homomorphic image of a polynomial ring over the regular ring $R$; note also that if $m \geq n$ then $S_{m} \Rightarrow S_{n}$. Hence, by $[7,(5.10 .9)]$, whatever the value of $n$ in (3), $B$ is equidimensional. Since $B$ is an integral extension of $R$, any two primes in $B$ having the same restriction to $R$ must have the same co-height in $B$ (see [10, Theorem 47]), and hence the same height in $B$. It follows from $[\mathbf{7},(5.7 .11)]$ that $B$ satisfies $S_{n}$ as a $B$-module if and only if it satisfies $S_{n}$ as an $R$-module.

THEOREM 2. Conjectures (2) and (3) are equivalent, and are implied by Conjecture (1).

Proof. First suppose that (3) holds, and let $R$ and $K$ be as in the statement of (2). Consider the ring $B=R \ltimes K$ formed by Nagata's process of idealization $[12$, p. 2]. Then $B$ is a local ring which dominates $R$ and $B$ is a finitely generated $R$-module. Since $B=R \oplus K$ as an $R$-module, $\operatorname{rank}_{R} B \leq j$. But as remarked in $[\mathbf{9}]$ (and as can easily be deduced from $[\mathbf{1 2}, \S 23]$ )

$$
e_{R}(B)=\operatorname{rank}_{R} B,
$$

where $e_{R}(B)$ is the multiplicity of $B$ as an $R$-module. By Auslander and Bridger's theorem $[\mathbf{1},(4.25)]$ (see $\left[5\right.$, Theorem 3.8]), $K$ satisfies $S_{j}$. Now as $R$-modules, $B_{P}=R_{P} \oplus K_{P}$ for all $P \in \operatorname{Spec} R$. By the definition of depth via the Ext-functor say (e.g. see $[5$, p. 3]), for all $P \in \operatorname{Spec} R$,

$$
\operatorname{depth}_{R} B_{P}=\min \left\{\operatorname{depth}_{R} R_{P}, \operatorname{depth}_{R} K_{P}\right\}=\min \{j, \text { height } P\},
$$

since $R$ is regular. Hence $B$ satisfies $S_{j}$ as an $R$-module, and so as a $B$-module, and $e_{R}(B) \leq j$. By (3) therefore, $B$ is Cohen-Macaulay. Thus $B$ is a free module, by $[12,(25.16)]$, and therefore $K$ is also free, by $[2$, p. 147, Ex. 4] (or [5, p. 25, Ex. 10]).

Conversely, suppose that (2) holds, and let $R$ and $B$ be as in (3). Now $B$ satisfies $S_{n}$ as an $R$-module, and by the remarks above, $\operatorname{rank}_{R} B \leq n$. Let $C=B / R$ and consider the exact sequence

$$
0 \rightarrow R \rightarrow B \rightarrow C \rightarrow 0
$$

Then $\operatorname{rank}_{R} C \leq n-1$.

Fix $P \in \operatorname{Spec} R$. Then we have the exact sequence

$$
0 \rightarrow R_{P} \rightarrow B_{P} \rightarrow C_{P} \rightarrow 0 \text {. }
$$


First suppose that height $P \leq n$. Then $B_{P}$ is a Cohen-Macaulay $R_{P}$-module, and $B_{P}$ is a semi-local integral over-ring of $R_{P}$. It easily follows that $B_{P}$ is itself a Cohen-Macaulay ring. Hence as before, by $[12,(25.16)]$ and $\left[2\right.$, p. 147, Ex. 4], $C_{P}$ is a free $R_{P}$-module and so, by the definition of depth via the Ext-functor (say) and by the regularity of $R, \operatorname{depth}_{R} C_{P}$ equals height $P$.

On the other hand, suppose that height $P>n$. It follows from the 'Depth Lemma' [5, p. 13], since $R$ is regular, that $\operatorname{depth}_{R} C_{P} \geq \operatorname{depth}_{R} B_{P}$ or $\operatorname{depth}_{R} C_{P}=$ height $P-1$. Whatever the case therefore, $\operatorname{depth}_{R} C_{P} \geq n$ since $B$ satisfies $S_{n}$ as an $R$-module. Hence $C$ also satisfies $S_{n}$ as an $R$-module, and $\operatorname{rank}_{R} C \leq n-1$. By (2) therefore, $C$ is free. Hence $B$ is free, and so is Cohen-Macaulay by $[\mathbf{1 2},(25.16)]$ again.

Finally consider Conjecture (1). Assume that it is valid. Then Conjecture (3) holds in the case where $R$ is a complete regular local ring, for it easily follows that in this case $B$ is also complete in the $\underline{m}^{\prime}$-adic (as well as the $\underline{m}$-adic) topology; by $\left[\mathbf{1 4}\right.$, Corollary 1, p. 299] and $[\mathbf{1 2},(23.6)] e_{R}(B) \geq e(B)$. By Theorem 2 (and its proof), Conjecture (2) then holds in the case where $R$ is complete. But by the faithful flatness of the completion functor, this is no restriction on Conjecture (2), which therefore holds as stated.

Corollary 1. Conjecture (3) holds when $\operatorname{dim} R(=\operatorname{dim} B) \leq 4$.

PRoOF. See the remarks after the statement of Conjectures (2) and (3).

If one wishes to dispense with the restriction that $R$ be a regular ring in Conjecture (3) and merely assume that $B$ is a complete local ring, then it easily follows (as on [3, p. 59], say) that $B$ is an equidimensional catenary ring which is a finitely generated torsion-free module over a complete local ring $S$ which is either regular or a hypersurface (i.e. a regular local ring modulo a principal ideal); moreover, $S$ contains a system of parameters for $B$. It is not clear (to me) what else can usefully be said about this more general situation. The example given on pp. 27-28 of $[\mathbf{8}]$ is of interest in this regard, as it shows that the analogue of $[\mathbf{1 2},(25.16)]$ for (complete) hypersurfaces (rather than regular local rings) is no longer valid.

NOTE ADDED IN PROOF. I would like to thank S. Ikeda for pointing out an error in the original version of this paper, and for very valuable discussions.

\section{REFERENCES}

1. M. Auslander and M. Bridger, Stable module theory, Mem. Amer. Math. Soc. 94 (1969).

2. N. Bourbaki, Commutative algebra, Addison-Wesley, Reading, Mass., 1972.

3. W. Bruns, On the number of elements independent with respect to an ideal, J. London Math. Soc. (2) 22 (1980), 57-62.

4. W. Bruns, E. G. Evans and P. Griffith, Syzygies, ideals of height two, and vector bundles, J. Algebra 67 (1980), 143-162.

5. E. G. Evans and P. Griffith, Syzygies, London Math. Soc. Lecture Notes Ser., vol. 106, Cambridge Univ. Press, Cambridge and New York, 1985.

6. W. Fulton, Introduction to intersection theory in algebraic geometry, CBMS Regional Conf. Ser. in Math., no. 54, Amer. Math. Soc., Providence, R. I., 1984.

7. A. Grothendieck, Éléments de géométrie algébrique, Inst. Hautes Études Sci. Publ. Math. 24 (1965).

8. M. Hochster, Contracted ideals from integral extensions of regular rings, Nagoya Math. J. 51 (1973), 25-43.

9. C. Huneke, A remark concerning multiplicities, Proc. Amer. Math. Soc. 85 (1982), 331332 . 
10. I. Kaplansky, Commutative rings, Allyn and Bacon, Boston, Mass., 1970.

11. H. Matsumura, Commutative algebra, Benjamin, New York, 1970.

12. M. Nagata, Local rings, Wiley-Interscience, New York, 1962.

13. D. G. Northcott, Lessons on rings, modules and multiplicities, Cambridge Univ. Press, London, 1968.

14. O. Zariski and P. Samuel, Commutative algebra. Vol. II, Springer-Verlag, New York, 1960.

Department of MAThematics, University of Edinburgh, James Clark MaXWELl BUILDING, King's BUILdings, MAYFIELD ROAD, EDINBURGH EH9 3JZ, SCOTLAND 